\title{
Jones-matrix diagnostics of the structure and classification of optical properties inherent to birefringent polycrystalline networks of human biological tissues
}

\author{
O.G. Ushenko, V.O. Balanetska, N.I. Zabolotna \\ Chernivtsi National University \\ 2, Kotsyubinsky vul., 58012 Chernivtsi, Ukraine
}

\begin{abstract}
Performed in this work is the complex statistical, correlation and fractal analysis of coordinate distributions of Jones-matrix elements corresponding to birefringent networks of liquid crystals in human biological liquids (saliva, blood plasma, bile). The authors have determined the values and ranges for changing statistical, correlation and spectral moments of the 1-st to 4-th orders that characterize Jones-matrix images of these objects. Ascertained are objective criteria for classification and differentiation of optical properties inherent to polycrystalline networks of biological crystals with various kinds of spatial symmetry: dendrite, spherolite and cluster ones.
\end{abstract}

Keywords: laser, polarization, birefringence, Jones matrix, biological liquids, statistical moments, autocorrelation, power spectrum, fractal.

Manuscript received 30.07.11; revised manuscript received 10.08.11; accepted for publication 14.09.11; published online 30.11.11.

\section{Introduction}

Among the methods for optical diagnostics of biological layers, the most widely spread are those of laser polarimetric diagnostics aimed at optical-anisotropic structure inherent to human tissues [1 - 31]. The main "information product" of these methods is obtaining the coordinate distributions for elements of Mueller and Jones matrixes corresponding to biological tissues (BT) [1 - 5] with the following statistical (statistical moments of the first to fourth orders [5, 6, 10, 14, 19, 25, 26, 30]), correlation (auto- and mutual-correlation functions [12, 17, 18, 21, 26]), fractal (fractal dimensionalities [5, 6, $25]$ ), singular (distributions of amounts of linear and circularly polarized states), wavelet (sets of wavelet coefficients for various scales of biological crystals [22, 28]) analyses. As a result, one can determine interrelations between a set of these parameters and distributions of optical axis directions as well as the birefringence value inherent to networks of optically single-axis protein (myosin, collagen, elastin, etc.) fibrils in optically-anisotropic component of BT layer. Being based on this approach, a large amount of methods for diagnostics and differentiation of pathological changes in BT structure that are related with their degenerativedystrophic as well as oncological changes [4 - 6, 12, 19, 20-22, 27, 29, 31].

It should be noted that there is a widely spread group of optically anisotropic biological objects, for which the methods of laser polarimetric diagnostics did not acquire wide application yet. Related to these objects are optically-thin (extinction coefficient $\tau \leq 0.1$ ) layers of various biological liquids (bile, urine, liquor, synovial liquid, blood plasma, saliva, etc.). These objects are considerably more accessible for direct laboratory analyses as compared with traumatic methods of BT biopsy. Being based on these reasons, it seems topical to adapt the methods of laser polarimetric diagnostics for studying the optically-anisotropic structure of BT polycrystalline networks.

Our work is aimed at searching the possibilities for diagnostics and classification of optical properties of human biological liquids with various spatial symmetry of polycrystalline networks. With this purpose, we offer to determine coordinate distributions corresponding to Jones-matrix elements with the following statistical, correlation and fractal analyses of these distributions.

\section{Main analytical relations}

Our analysis of experimental results is based on the following conceptions developed for optically anisotropic protein fibrils [1 - 4, 7, 9, 14, 16, 23-27, 30]:

- $\quad$ separate (partial) amino acid crystals are optically uniaxial and birefringent;

- $\quad$ optical properties of a partial crystal are exhaustively full described with the Jones operator [5] 
$\{J\}=\left\|\begin{array}{ll}J_{11} & J_{12} \\ J_{21} & J_{22}\end{array}\right\|=\left\|\begin{array}{ll}\cos ^{2} \rho+\sin ^{2} \rho \exp (-i \delta) ; & \cos \rho \sin \rho[1-\exp (-i \delta)] \\ \cos \rho \sin \rho[1-\exp (-i \delta)] ; & \sin ^{2} \rho+\cos ^{2} \rho \exp (-i \delta) ;\end{array}\right\|$.

Here, $\rho$ is the direction of the optical axis; $\delta=2 \pi / \lambda \Delta n d-$ phase shift between orthogonal components $E_{x}$ and $E_{y}$ of the amplitude of illuminating laser wave with the wavelength $\lambda ; \Delta n$ birefringence index for the crystal with geometric dimension $d$.

The Jones matrix $\{\mathfrak{\Im}\}$ of a flat layer corresponding to polycrystalline network can be defined as a sum of operators $\{J\}_{l}$ for $K$ separate amino acid crystal $[5,11,30]$

$$
\{\mathfrak{J}\}=\sum_{l=1}^{K}\{J\}_{l},
$$

It can be shown that for $Q$ sequentially placed flat layers the net Jones matrix is defined with the expression $\{\mathfrak{N}\}=\prod_{q=1}^{Q}\{\mathfrak{I}\}_{q}=\{\mathfrak{J}\}_{Q} \times\{\mathfrak{I}\}_{Q-1} \times \ldots \times\{\mathfrak{I}\}_{2} \times\{\mathfrak{I}\}_{1}$.

In an expanded version, the matrix elements $\aleph_{i k}$ possess a cumbersome analytical look. Therefore, to consider the structure of generalized Jones matrix $\{\boldsymbol{N}\}$ in more convenient manner, we limit ourselves (without losses in fullness of analysis) by the case of bilayer $(Q=2)$ polycrystalline network. With account of this approximation as well as an evident look of dependences (relation (1)) for matrix elements $J_{i k}(\rho, \delta)$, it is possible to show that the elements of generalized Jones matrix can be expressed with the following dependences

$\aleph_{i k}=R_{i k}(\rho)+T_{i k}(\rho) \exp (-i \delta)+$

$+U_{i k}(\rho) \exp (-2 i \delta)$.

Here, $R_{i k} ; T_{i k} ; U_{i k}$ are the coefficients expressed via quasi-harmonic functionals ( $\left.\cos ^{2} " ; " \sin ^{2} " ; " \cos -\sin "\right)$ from coordinate changes in orientations of optical axis $\rho(x, y)$

$$
\begin{aligned}
& \left\{\begin{array}{l}
R_{11}=\cos ^{2} \rho \cos ^{2}(\rho+\Delta \rho)+(0,5)^{4} \sin 2 \rho \sin 2(\rho+\Delta \rho) ; \\
T_{11}=\left[\cos ^{2} \rho \sin ^{2}(\rho+\Delta \rho)+\sin ^{2} \rho \cos ^{2}(\rho+\Delta \rho)-2 \sin 2 \rho \sin 2(\rho+\Delta \rho)\right] \exp (-i \delta) ; \\
U_{11}=\left[\sin ^{2} \rho \sin ^{2}(\rho+\Delta \rho)+2 \sin 2 \rho \sin 2(\rho+\Delta \rho)\right] \exp (-2 i \delta) .
\end{array}\right. \\
& \left\{\begin{array}{l}
R_{12 ; 21}=0,25\left[\cos ^{2} \rho \sin 2(\rho+\Delta \rho)+\sin 2 \rho \cos ^{2}(\rho+\Delta \rho)\right] \\
T_{12 ; 21}=0,25\left[\sin 2 \rho \cos ^{2}(\rho+\Delta \rho)+\sin ^{2} \rho \sin 2(\rho+\Delta \rho)\right] \exp (-i \delta) ; \\
U_{12 ; 21}=0,25\left[\sin ^{2} \rho \sin 2(\rho+\Delta \rho)+\sin 2 \rho \cos ^{2} \rho\right] \exp (-i 2 \delta) .
\end{array}\right. \\
& \left\{\begin{array}{l}
R_{22}=\sin ^{2} \rho \sin ^{2}(\rho+\Delta \rho)+(0,5)^{4} \sin 2 \rho \sin 2(\rho+\Delta \rho) ; \\
T_{22}=\left[\sin ^{2} \rho \cos ^{2}(\rho+\Delta \rho)+\cos ^{2} \rho \sin 2(\rho+\Delta \rho)-2 \sin 2 \rho \sin 2(\rho+\Delta \rho)\right] \exp (-i \delta) ; \\
U_{22}=\left[\cos ^{2} \rho \cos ^{2}(\rho+\Delta \rho)+2 \sin 2 \rho \sin 2(\rho+\Delta \rho)\right] \exp (-2 i \delta) .
\end{array}\right.
\end{aligned}
$$


As a base for analyzing the coordinate structure of $\left(\aleph_{i k}\right)_{j}$ distributions, we took the autocorrelation method with using the function $[12,21,26]$

$G(\Delta x)=\frac{1}{X_{0}} \int_{1}^{X_{0}}\left[\aleph_{i k}(x)\right]\left[\aleph_{i k}(x-\Delta x)\right] d x$

Here, $\Delta x$ is the "step" of changing the coordinates $x=1 \div X_{0}$.

As parameters that characterize the dependences $G(\Delta x)$, we chose the set of correlation moments of the 1-st to 4-th orders $K_{l=1 ; 2 ; 3 ; 4}$ that are defined like to relations (8).

Estimating the degree of self-similarity and repeatability for different geometric $(d)$ scales of the structure inherent to coordinate distributions of elements $\aleph_{i k}$ corresponding to the Jones matrix of polycrystalline networks was performed by calculation of logarithmic dependences for power spectra $\log J\left(\aleph_{i k}\right)-\log \left(d^{-1}\right)$ that were approximated using the least-squares method to the curves $\Phi(\eta)$. For the straight parts of the curves $\Phi(\eta)$, determined are the slope angles $\eta_{i}$ and calculated are the values of fractal dimensionalities for the sets of $\aleph_{i k}$ values by using the relations $[5,6,11,25]$

$D_{i}(g)=3-\operatorname{tg}_{i}$.

Classification of coordinate distributions for matrix elements $\aleph_{i k}(x, y)$ was carried out in accord with the criteria offered in [5]. If the value of the slope angle $\eta=$ const in the dependences $\Phi(\eta)$ for 2 or 3 decades of changing the sizes $d$, then the distributions $\aleph_{i k}(x, y)$ are fractal. Under condition that several constant slope angles are available in the curve $\Phi(\eta)$, the sets $\aleph_{i k}(x, y)$ are multi-fractal. When no stable slope angles are available over the whole interval of changing the sizes $d$, the sets $\aleph_{i k}(x, y)$ are considered as random.

To make this comparative analysis of $\log J\left(\aleph_{i k}\right)-\log \left(d^{-1}\right)$ dependences more objective, let us introduce the conception of spectral moments from the 1-st to 4-th orders $S_{j=1 ; 2 ; 3 ; 4}$ - the relation (8).

\section{Brief characterization of the studied objects}

As the studied objects, we used smears of biological liquids taken from healthy patients:

- $\quad$ saliva (21 samples) - group 1;

- $\quad$ blood plasma (21 samples) - group 2;

- $\quad$ bile (21 samples) - group 3.

This choice of biological liquids was related with the following factors:

- they comprise a wide circle of physiological functions in a human organism;

- diverse biochemical structure of these liquids, as well as others no less important from the physiological viewpoint (transudate, exudate, .......... liquor, ....), possesses common optical properties that are related with birefringent liquidcrystalline networks of amino acids, calcium bilirubinate, fat acids, cholesterol monohydrate, etc.

Fig. 1 shows polarization-visualized images of polycrystalline networks inherent to birefringent biological crystals of the liquid samples in all the groups. These images were obtained for crossed transmission planes of the polarizer and analyzer.

The comparative analysis of laser images corresponding to smears of human liquids enabled us to ascertain:

- $\quad$ availability of optically-anisotropic polycrystalline networks inherent to biological crystals in all the groups of liquids;

- dependence of optical anisotropy parameters (distribution of orientation directions for biological crystals and the value of their birefringence) on the type of liquid (Fig. 1, fragments a, b, c);

- in the geometrical structure of polycrystalline networks typical for saliva (a) and blood plasma (b), there predominant are needle-like or dendritelike structures, while for bile (c) - spherolitic creations.

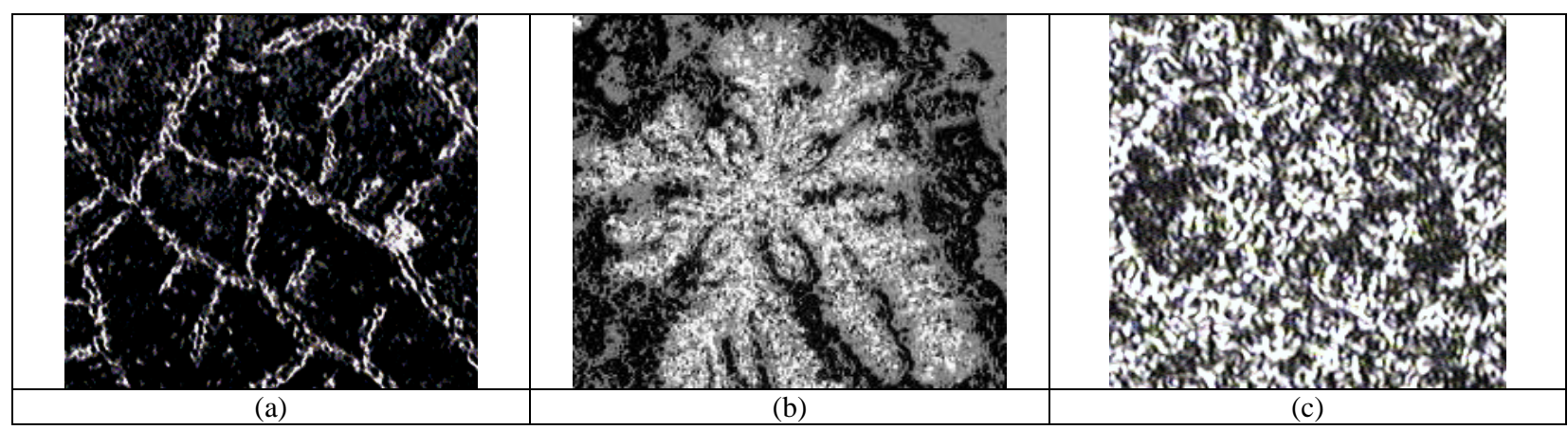

Fig. 1. Polarization-visualized laser images corresponding to polycrystalline networks of saliva (a), blood plasma (b) and bile (c).

(C) 2011, V. Lashkaryov Institute of Semiconductor Physics, National Academy of Sciences of Ukraine 


\section{Optical setup for Jones-matrix mapping the optically-anisotropic biological liquids}

Shown in Fig. 2 is the optical scheme of a polarimeter for measuring the coordinate distributions of Jonesmatrix elements corresponding to birefringent layers.

Illumination was provided with a parallel $\left(\varnothing=10^{4} \mu \mathrm{m}\right)$ beam of a He-Ne laser $(\lambda=0.6328 \mu \mathrm{m}, \mathrm{W}$ $=5.0 \mathrm{~mW}$ ). The polarization illuminator consists of the quarter-wave plates 3,5 and polarizer 4 , which provides formation of a laser beam with an arbitrary polarization state. Using the micro-objective 7 (magnification 4x), images of biological layers were projected onto the plane of light-sensitive area (800x600 pixels) of the CCDcamera 10 that provided measurements of structural elements within the range 2 to $2000 \mu \mathrm{m}$. The analysis of laser images was performed using the polarizer 9 and quarter-wave plate 8 .

Conditions of our experiments were chosen in such a manner that enabled to practically eliminate spatialangle aperture filtration when forming the images of biological liquids. It was provided by coincidence of angular characteristics for indicatrixes of light scattering by samples of biological liquids ( $\Omega \approx 16^{0}$ ) and angular aperture of the used micro-objective $\left(\Delta \omega=20^{\circ}\right)$. Here, $\Omega$ is the angular cone of indicatrixes where $98 \%$ of all the scattered radiation energy is concentrated.

\section{Analysis of experimental results and their discussion}

Adduced in Fig. 3 are coordinate (a, b, c), probability (d, e, f), autocorrelation (g, h, i), spectral $(k, l, m)$ dependences that characterize Jones-matrix images of $\aleph_{11}(m \times n)$. elements for optically-thin (extinction coefficient $\tau \leq 0.01$ ) layers of saliva (a, d, g, k), blood plasma (b, e, h, l) and bile (c, f, i, m).

Performing the model analysis (relations (1) to (7)), we have shown that our choice of elements $\aleph_{11}(\rho, \delta)$ in the Jones matrix allows estimating (without lowering the fullness of this analysis) the contribution of orientationphase $(\rho, \delta)$ structure of polycrystalline networks with different symmetry (Fig. 1) to formation of their polarization properties.

The results obtained in the course of studying the Jones-matrix images of $\aleph_{11}(m \times n)$. element, which characterize polarization properties of the orientationphase structure inherent to polycrystalline networks (Fig. 1) of optically-thin layers for biological liquids of different types, are indicative of a sufficient level of birefringence in these liquid crystals. This fact is confirmed by a wide range of changes $\left(0 \leq \Delta \aleph_{11} \leq 1\right)$ in intrinsic values of the given matrix element $\aleph_{11}(\rho, \delta)$. On the other hand, different spatial symmetry of distributions for optical axis directions $(\rho(m \times n))$ is pronounced in individual dependences for the series of histograms $H\left(\aleph_{11}\right)$ corresponding to the probability of element values in the Jones matrix (Fig. 3, fragments (d, e, f). For instance, in the case of dendrite network of biological crystals in saliva layer, the histogram $H\left(\aleph_{11}\right)$ is asymmetric relatively to the main extreme, the value of which is $2-7$ times higher than those of other extremes in the probability of local values $\aleph_{11}(\rho, \delta)$ (Fig. 3, fragment (d)). This fact is in a good correlation with model notions about the influence of distributions describing the directions of optical axes for partial crystals that form birefringent network (relations (1) to (7), Fig. 1, fragment (a)) on the coordinate structure of the Jones-matrix image $\aleph_{11}(m \times n)$.

Azimuth symmetry of optical axis directions for partial crystals in spherolite polycrystalline network of albumin and globulin in optically-thin layer of blood plasma (Fig. 1, fragment (b)) is pronounced in more uniform distribution for extreme values in the histogram $\mathrm{H}\left(\aleph_{11}\right)$ over the whole range of changes in local values of the matrix element $\aleph_{11}(\rho, \delta)$ (Fig. 3, fragment (e)).

Polarization properties of cluster polycrystalline component in the bile layer (Fig. 1, fragment (c)) are expressed in rather symmetrical, close to the level of probabilistic one, distribution for extremes of the histogram $\mathrm{H}\left(\aleph_{11}\right)$ describing probabilities of random values $\aleph_{11}(\rho, \delta)$ (Fig. 3 , fragment (f)).

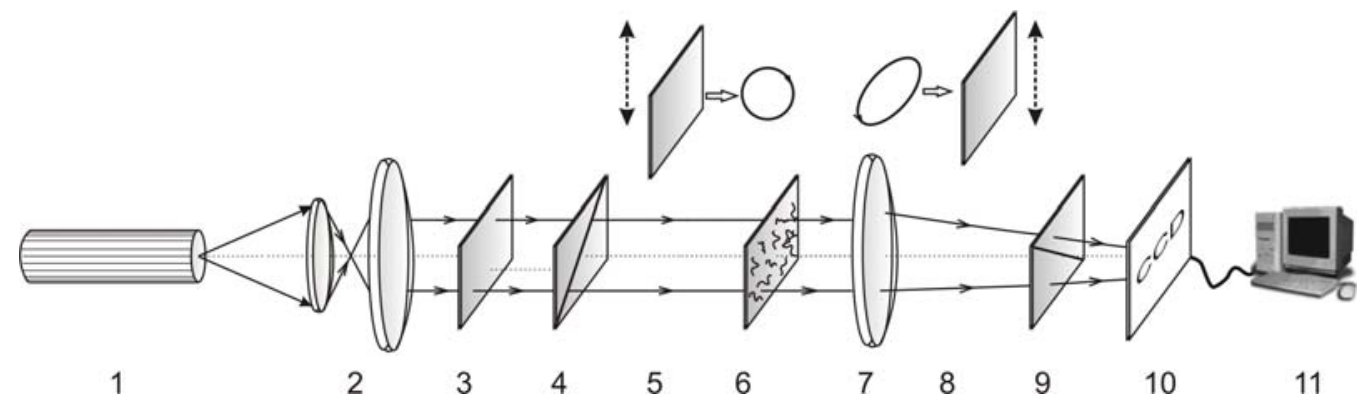

Fig. 2. Optical scheme of the polarimeter: 1 - He-Ne laser; 2 - collimator; 3 - stationary quarter-wave plate; 5 , 8 - mechanically movable quarter-wave plates; 4 , 9 - polarizer and analyzer, respectively; 6 - object under investigation; 7 - micro-objective; 10 - CCD camera; 11 - personal computer. 
The correlation approach (relation (9)) to analyses of $\aleph_{11}(m \times n)$ elements in Jones-matrix images for various types of biological liquids has shown that, in the case of dendrite and spherolite birefringent networks, the autocorrelation functions $G_{11}(\Delta x)$ are decaying dependences with clearly pronounced fluctuations of intrinsic values (Fig. 3, fragments (g, h)). The experimentally found fact on availability of harmonic modulation is in good accordance with analytical data (relation (1) to (7)) for the influence of orientation-phase ( $\rho, \delta$ ) parameters corresponding to optically uniaxial birefringent crystals on distributions $\aleph_{11}(\rho, \delta)$ that are superposition of various harmonic components $\left(\sim " \cos ^{2} " ; " \sin ^{2} " ; " \cos -\sin "\right) . \quad$ The autocorrelation

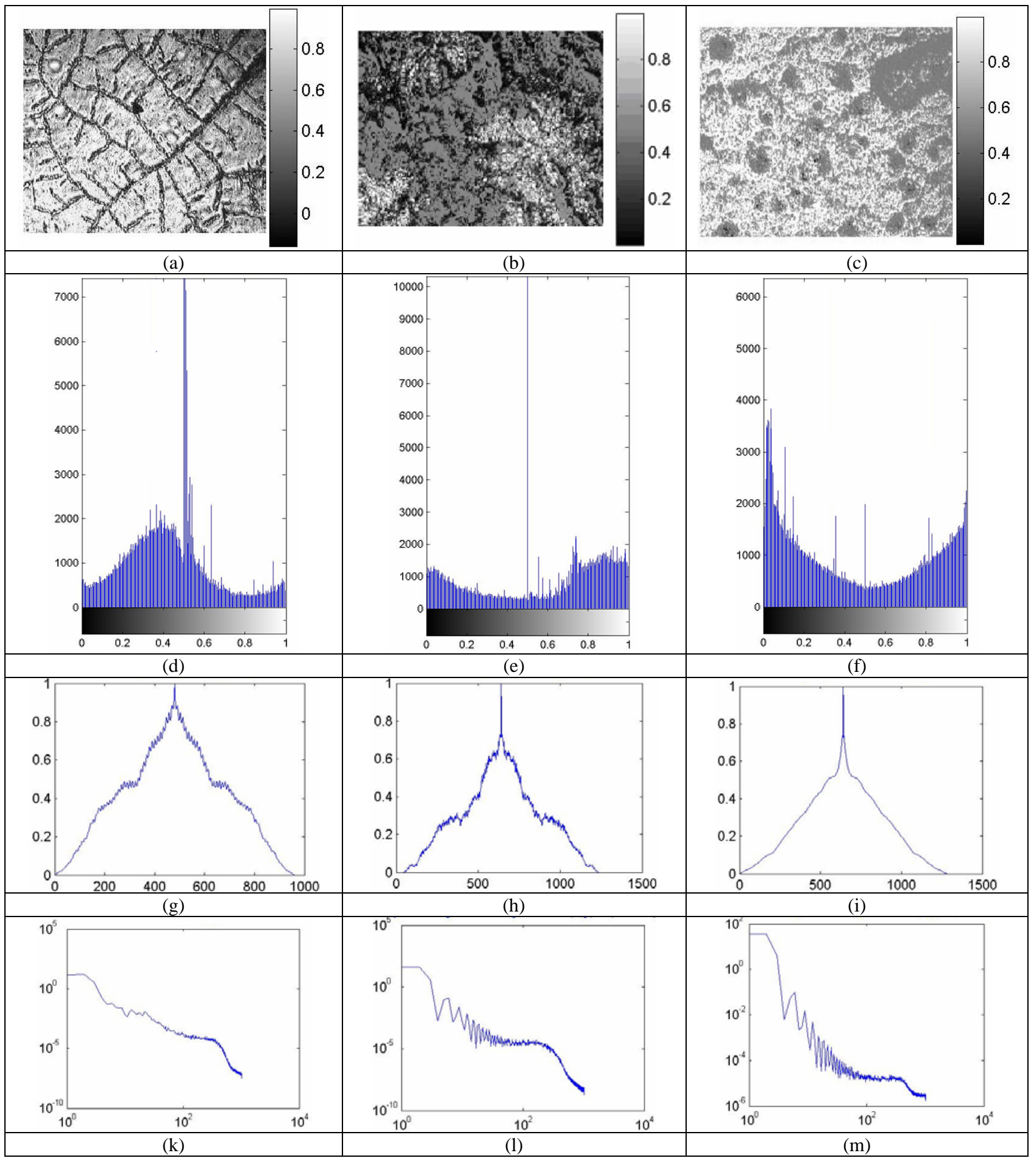

Fig. 3. Statistical, correlation and fractal parameters of Jones-matrix images $\aleph_{11}(m \times n)$. for optically-thin layers of saliva (a, d, g, k), blood plasma (b, e, h, l) and bile (c, f, i, m). See explanations in the text. 
functions $G_{11}(\Delta x)$ for Jones-matrix images $\aleph_{11}(m \times n)$ corresponding to the cluster birefringent network of bile samples from the group 3 are monotonous decaying dependences without any fluctuations of intrinsic values (Fig. 3, fragment (i)), which can be related with practically full disordering the directions of optical axes in optically anisotropic structures.

Discrete scale-repeated changes in orientations of optical axes of partial biological crystals (Fig. 1, fragments (a, b, c)) with simultaneous multiple changes in the phase $\delta$ period (relations (4) to (7)) is pronounced as formation of self-similar coordinate distributions of Jones-matrix elements $\aleph_{11}(\rho, \delta)$ in the plane of biological liquid layers in all the groups. It is seen from the analysis of logarithmic dependences $\log J\left(\aleph_{11}\right)-\log \left(d^{-1}\right)$ (Fig. 3, fragments $(\mathrm{k}, \mathrm{l}, \mathrm{m})$ ) that the approximating curves $\Phi(\eta)$ are broken lines with three slope angles. In other words, distributions of matrix element values $\aleph_{11}(m \times n)$ are multi-fractal.

The results of our quantitative analysis concerning values and ranges for changing the statistical, correlation and spectral moments in coordinate distributions of $\aleph_{11}(m \times n)$ that characterize polarization properties of birefringent networks corresponding to biological liquids of various types (Fig. 1) are summarized in Table 1.

The analysis of the obtained data enabled us to find satisfactory correlation with the results of computer modeling, and it shows that the whole set of statistical, correlation and spectral moments of the 1-st to 4-th orders characterizing coordinate distributions of Jonesmatrix elements possesses individual sets of values dependent on optical-and-geometrical parameters corresponding to amino acid polycrystalline networks. For instance, changes in spatial symmetry of partial amino acid crystals, in accord with the scenario "dendrite network - spherolitic network - cluster ensemble”, is pronounced as:

Table 1. Statistical $(M ; \sigma ; A ; E)$, correlation $\left(K_{i=1 ; 2 ; 3 ; 4)}\right.$ and spectral $\left(S_{i=1 ; 2 ; 3 ; 4)}\right.$ parameters of Jones-matrix images $\aleph_{11}(m \times n)$ inherent to polycrystalline networks of biological liquids

\begin{tabular}{|c|c|c|c|}
\hline Parameters & $\begin{array}{c}\text { Saliva (21 } \\
\text { samples) }\end{array}$ & $\begin{array}{l}\text { Blood } \\
\text { plasma (21 } \\
\text { samples) }\end{array}$ & $\begin{array}{l}\text { Bile (21 } \\
\text { samples) }\end{array}$ \\
\hline$M$ & $0.41 \pm 0.19$ & $0.49 \pm 0.14$ & $0.54 \pm 0.12$ \\
\hline$\sigma$ & $0.24 \pm 0.092$ & $0.28 \pm 0.089$ & $0.34 \pm 0.12$ \\
\hline$A$ & $1.26 \pm 0.41$ & $0.77 \pm 0.072$ & $0.45 \pm 0.097$ \\
\hline$E$ & $3.17 \pm 0.075$ & $1.89 \pm 0.48$ & $0.89 \pm 0.13$ \\
\hline$K_{1}$ & $0.41 \pm 0.086$ & $0.44 \pm 0.098$ & $0.47 \pm 0.105$ \\
\hline$K_{2}$ & $0.09 \pm 0.0082$ & $0.15 \pm 0.029$ & $0.25 \pm 0.062$ \\
\hline$K_{3}$ & $3.28 \pm 0.71$ & $1.91 \pm 0.46$ & $0.89 \pm 0.23$ \\
\hline$K_{4}$ & $5.88 \pm 1.44$ & $3.08 \pm 0.57$ & $1.86 \pm 0.32$ \\
\hline$S_{1}$ & $0.51 \pm 0.13$ & $0.44 \pm 0.072$ & $0.38 \pm 0.16$ \\
\hline$S_{2}$ & $0.27 \pm 0.061$ & $0.14 \pm 0.031$ & $0.11 \pm 0.014$ \\
\hline$S_{3}$ & $0.69 \pm 0.11$ & $0.34 \pm 0.067$ & $0.18 \pm 0.11$ \\
\hline$S_{4}$ & $1.25 \pm 0.24$ & $0.64 \pm 0.17$ & $0.29 \pm 0.08$ \\
\hline
\end{tabular}

- $\quad$ insignificant growth of the mean $M$ (by $1.2-1.3$ times) and dispersion $\sigma$ (by $1.2-1.4$ times) and, contrary, considerable decrease in values of statistical moments of the 3-rd ( $A$ - by $1.6-2.8$ times) and 4-th orders ( $E$ - by $1.7-3.6$ times) of coordinate distributions for $\aleph_{11}(m \times n)$ elements;

- decaying oscillations of autocorrelation functions corresponding to Jones-matrix images as well as in decrease in respective values of correlation moments of higher orders $\left(K_{3}\right.$ - by 1.7 - 3.7 times and $K_{4}$ - by 1.9 - 3.1 times) for these dependences, while the correlation moment of the 2-nd order $K_{2}$ is $1.7-2.8$ times increased;

- decrease by $1.3-4.3$ times in the whole set of spectral moments of the 1-st to 4 -th orders $S_{i=1 ; 2 ; 3 ; 4}$ that characterize logarithmic dependences $\log J\left(\aleph_{11}\right)-\log \left(d^{-1}\right)$.

Fig. 4 shows circle diagrams for the values of ranges $\Delta Z^{(i)}$ corresponding to changes in values of the set comprising statistical $(M ; \sigma ; A ; E)$, correlation $\left(K_{i=1 ; 2 ; 3 ; 4}\right)$ and spectral ( $\left.S_{i=1 ; 2 ; 3 ; 4}\right)$ parameters of Jonesmatrix images $\aleph_{11}(m \times n)$ inherent to polycrystalline networks of human biological liquids.

Fig. 4. On the analysis of capabilities typical for Jonesmatrix classification of polarization properties inherent to polycrystalline networks in human biological liquids.

Classification dependences were plotted in the following manner. In the Cartesian coordinate system, the abscissa corresponds to values of ranges $\Delta Z^{(i)}=Z_{\max }^{(i)}-Z_{m s n}^{(i)}$, while the ordinate - to the values of parameters $Z_{i=1 ; 2 ; 3 ; 4}$. For every group of human biological liquids, we determined the dispersion $Z^{(2)}=\Delta Z$ that served as a radius of the circle diagram $Z^{(i)}(\Delta Z)$ of random values related to parameters $Z_{i=1 ; 2 ; 3 ; 4}$ characterizing the Jones-matrix images $\aleph_{11}(m \times n)$ of biological liquid polycrystalline networks. The center of this circle diagram was determined as the cross-point of two normals to axes of the chosen coordinate system, which were plotted from the middles of the segments $Z^{(i)}$ and $\Delta Z$.

It is seen from the results adduced in Fig. 4 that the ranges for changing $\Delta Z$ values for statistical moments of various orders $M, \sigma, A, E$ possess individual character, and can be used in various ways to classify the studied types of biological liquids. For example, geometrical areas of the circles $M(\Delta Z)$ and $\sigma(\Delta Z)$ determined for Jones-matrix images $\aleph_{11}(m \times n)$ within the limits of various biological liquid groups possess a high (up to 90\%) level of superposition (Fig. 4, fragments (a, b)). Considerably larger differences (Fig. 4, fragments (c, d)) are observed when comparatively 
analyzing the dependences $A(\Delta Z)$ and $E(\Delta Z)$. The superposition level in the case of circle diagrams for the 3-rd statistical moment is close to 25 - 35\%, for the 4-th $-20-25 \%$.

The respective diagrams $K_{2}(\Delta z), \quad K_{3}(\Delta z)$ and $K_{4}(\Delta z)$ for parameters of autocorrelation dependences $G_{11}(\Delta x)$ corresponding to Jones-matrix images $\aleph_{11}(m \times n)$ are not practically overlapped (Fig. 4, fragments (f, g, h)).

As to the set of spectral statistical moments $S_{i=2 ; 3 ; 4}$, we have ascertained the possibility to efficiently differentiate the polarization properties of dendrite, spherolite and cluster optically anisotropic networks the diagrams $S_{2}(\Delta z), \quad S_{3}(\Delta z)$ and $S_{4}(\Delta z)$ are not overlapped (Fig. 4, fragments (k, l, m)).

\section{Conclusions}

1. Offered in this work is the method of superposition of Jones matrixes to describe polarization properties of birefringent polycrystalline networks inherent to human biological liquids.

2. It has been ascertained that the Jones-matrix images corresponding to polycrystalline layers of human biological liquids with different types of spatial symmetry possess individual values of statistical, correlation and spectral moments of the 2-nd to 4-th orders.

3. Found and grounded is the complex of criteria for Jones-matrix diagnostics and classification of optical properties inherent to birefringent dendrite, spherolite and cluster polycrystalline networks of human biological liquids.

\section{References}

1. W.-F. Cheong, S. A. Prahl, A. J. Welch, "A Review of the Optical Properties of Biological Tissues," IEEE J. Quantum Electron, Vol. 26, pp. 21662185, Dec. 1990.

2. S. A. Prahl, M. Keijzer, S. L. Jacques, A. J. Welch, "A Monte Carlo model of light propagation in tissue," SPIE Proceedings of Dosimetry of Laser Radiation in Medicine and Biology, Vol. IS 5, pp. 102-111, 1989.

3. E. Wolf, "Unified theory of coherence and polarization of random electromagnetic beams ," Phys. Lett. A., Vol. 312, pp. 263-267, 2003.

4. Alexander G. Ushenko and Vasilii P. Pishak, "Laser Polarimetry of Biological Tissue: Principles and Applications", in Handbook of CoherentDomain Optical Methods: Biomedical Diagnostics, Environmental and Materials Science, vol. I, Valery V. Tuchin, Ed. Boston: Kluwer Academic Publishers, 2004, pp. 93-138.

5. O. V. Angelsky, A. G. Ushenko, Yu. A. Ushenko,
V. P. Pishak, "Statistical and Fractal Structure of Biological Tissue Mueller Matrix Images", in Optical Correlation Techniques and Applications, Oleg V. Angelsky, Ed. Washington: Society of Photo-Optical Instrumentation Engineers , 2007, pp. 213-266.

6. O.V. Angelsky, A.G. Ushenko, Yu.A. Ushenko, V.P. Pishak, and A.P. Peresunko, "Statistical, Correlation, and Topological Approaches in Diagnostics of the Structure and Physiological State of Birefringent Biological Tissues", in Handbook of Photonics for Biomedical Science, Valery V. Tuchin, Ed. USA: CRC Press, 2010, pp. 21-67.

7. Alexander G. Ushenko, "Polarization structure of laser scattering fields," Optical Engineering, vol. 34(4), pp. 1088-1093, November 1995.

8. A.G. Ushenko, "Laser diagnostics of biofractals," Quantum Electronics, vol. 29(12), pp. 1078-1084, December 1999.

9. O.V. Angel'skii, A.G. Ushenko, A.D. Arkhelyuk, S.B. Ermolenko, D.N. Burkovets, "Structure of matrices for the transformation of laser radiation by biofractals,” Quantum Electronics, vol. 29(12), pp. 1074-1077, December 1999.

10. O.V. Angel'skii, A.G. Ushenko A.D. Arheluk, S.B. Ermolenko, D. N. Burkovets, "Scattering of Laser Radiation by Multifractal Biological Structures," Optics and Spectroscopy, vol. 88(3), pp. 444-448, March 2000.

11. A.G. Ushenko, "Polarization Structure of Biospeckles and the Depolarization of Laser Radiation,” Optics and Spectroscopy, vol. 89(4), pp. 597-601, October 2000.

12. A.G. Ushenko, "Stokes-correlometry of biotissues,” Laser Physics, vol. 10(5), pp.12861292, May 2000.

13. A.G. Ushenko, "The Vector Structure of Laser Biospeckle Fields and Polarization Diagnostics of Collagen Skin Structures," Laser Physics, vol. 10(5), pp. 1143-1149, May 2000.

14. A.G. Ushenko, "Laser polarimetry of polarizationphase statistical moments of the object field of optically anisotropic scattering layers," Optics and Spectroscopy, vol. 91(2), pp. 313-316, February 2001.

15. A.G. Ushenko, "Polarization contrast enhancement of images of biological tissues under the conditions of multiple scattering," Optics and Spectroscopy, vol. 91(6), pp. 937-940, August 2001.

16. A.G. Ushenko, "Laser probing of biological tissues and the polarization selection of their images," Optics and Spectroscopy, vol. 91(6), pp.932-936, August 2001.

17. A.G. Ushenko, "Correlation processing and wavelet analysis of polarization images of biological tissues," Optics and Spectroscopy, vol. 91(5), pp.773-778, June 2002. 
18. A.G. Ushenko, "Polarization correlometry of angular structure in the microrelief pattern or rough surfaces," Optics and spectroscopy, vol. 92(2), pp. 227-229, June 2002.

19. O.V. Angelsky, A.G. Ushenko, Ye.G. Ushenko, "2D Stokes Polarimetry of Biospeckle Tissues Images in Pre-Clinic Diagnostics of Their Pre-Cancer States,” Journal of Holography and Speckle, vol. 2(1), pp. 26-33, April 2005.

20. Oleg V. Angelsky, Alexander G. Ushenko, and Yevheniya G. Ushenko, "Complex degree of mutual polarization of biological tissue coherent images for the diagnostics of their physiological state,” J. Biomed. Opt., vol. 10(6), Article ID 060502, November 2005.

21. O. V. Angelsky, A. G. Ushenko, and Ye. G. Ushenko, "Investigation of the correlation structure of biological tissue polarization images during the diagnostics of their oncological changes," Physics in Medicine and Biology, vol. 50(20), pp. 48114822, September 2005.

22. Oleg V. Angelsky, Alexander G. Ushenko, Yevheniya G. Ushenko, Yuriy Y. Tomka, "Polarization singularities of biological tissues images,” J. Biomed. Opt., vol. 11(5), Article ID 054030, September-October 2006.

23. O.G. Ushenko, S.G. Guminetsky, A.V. Motrich, "Optical properties of urine, blood plasma and pulmonary condensate of the patients with pulmovnary form of tuberculosis," Photoelectronics, vol.16, pp. 133-139, June 2007.

24. S.H. Guminetskiy, O.G. Ushenko, I.P. Polyanskiy, A.V. Motrych, F.V. Grynchuk, "The optical method for investigation of the peritonitis progressing process," Proceedings of the SPIE, vol. 7008, Article ID 700827, April 2008.

25. Alexander Ushenko, Sergej Yermolenko, Alexander Prydij, Stepan Guminetsky, Ion Gruia, Ovidiu Toma, Konstantin Vladychenko, "Statistical and fractal approaches in laser polarimetry diagnostics of the cancer prostate tissues," Proceedings of the SPIE, vol. 7008, Article ID 70082C, April 2008.
26. A.G. Ushenko, A.I. Fediv, Yu.F. Marchuk, "Correlation and fractal structure of Jones matrices of human bile secret," Proceedings of the SPIE, vol. 7368, Article ID 73681Q, July 2009.

27. A.G. Ushenko, Yu.Ya. Tomka, V.I. Istratiy, "Polarization selection of two-dimensional phaseinhomogeneous birefringence images of biotissues," Proceedings of the SPIE, vol. 7388, Article ID 73881L, December 2009.

28. A.G. Ushenko, A.I. Fediv, Yu.F. Marchuk, "Singular structure of polarization images of bile secret in diagnostics of human physiological state," Proceedings of the SPIE, vol. 7368, Article ID 73681S, July 2009.

29. S.B. Yermolenko, A.G. Ushenko, P. Ivashko, "Spectropolarimetry of cancer change of biotissues," Proceedings of the SPIE, vol. 7388, Article ID 73881D, December 2009.

30. A.G. Ushenko, I. Z.Misevich, V. Istratiy, I. Bachyns'ka, A. P. Peresunko, Omar Kamal Numan, and T. G. Moiysuk, "Evolution of Statistic Moments of 2D-Distributions of Biological Liquid Crystal Net Mueller Matrix Elements in the Process of Their Birefringent Structure Changes,” Advances in Optical Technologies, vol. 2010, Article ID 423145, March 2010.

31. O. V. Dubolazov, A. G. Ushenko, V. T. Bachynsky, A. P. Peresunko, and O. Ya. Vanchulyak, "On the Feasibilities of Using the Wavelet Analysis of Mueller Matrix Images of Biological Crystals,” Advances in Optical Technologies, vol. 2010, Article ID 162832, March 2010. 